\title{
Cimento de alfa-fosfato tricálcico de dupla pega no preenchimento de cavidade anoftálmica - estudo clínico
}

\author{
Double-setting $\alpha$-tricalcium phosphate cement for the completion \\ of anophthalmic cavity - clinical study
}

\author{
Arianne Pontes ORIÁ ${ }^{1}$; Francisco de Assis DÓREA NETO²; Luis Alberto dos SANTOS ${ }^{3}$; \\ José Luiz LAUS ${ }^{4}$; Deusdete Conceição GOMES JUNIOR ${ }^{5}$ \\ ${ }^{1}$ Universidade Federal da Bahia (UFBA), Escola de Medicina Veterinária e Zootecnia, Salvador - BA, Brasil \\ ${ }^{2}$ União Metropolitana de Educação e Cultura (UNIME), Salvador - BA, Brasil \\ ${ }^{3}$ Universidade Federal do Rio Grande do Sul (UFRGS), Porto Alegre - RS, Brasil \\ ${ }^{4}$ Universidade Estadual Paulista (UNESP), Jaboticabal - SP, Brasil \\ ${ }^{5}$ Universidade Federal da Bahia (UFBA), Salvador - BA, Brasil
}

\begin{abstract}
Resumo
Foi avaliada a conveniência do emprego do cimento de alfa-fosfato tricálcico de dupla pega como implante para o preenchimento de cavidade anoftálmica de cães. Os animais foram provenientes do Serviço de Oftalmologia do Hospital Veterinário, da Universidade Estadual Paulista - Câmpus de Jaboticabal e de uma clínica privada. O trabalho foi realizado em dois anos. A idade dos pacientes oscilou entre 2 a 11 anos e o peso de 9 a $50 \mathrm{~kg}$. Os implantes variaram de 19 a $25 \mathrm{~mm}$ de diâmetro. O edema local foi observado em todos os animais durante o pós-operatório imediato. Das dez cirurgias realizadas em sete animais, em nove (90\%) o resultado estético foi satisfatório, enquanto em um (10\%) foi insatisfatório, devido a complicações que levaram à remoção do implante. A utilização do cimento de alfa-fosfato tricálcico de dupla pega no preenchimento de cavidade anoftálmica mostrou-se factível e é uma alternativa que possibilita boa aparência estética após a remoção do bulbo do olho.
\end{abstract}

Palavras-chave: Hidroxiapatita. Enucleação ocular. Olho artificial.

\begin{abstract}
The double-setting alpha-tricalcium phosphate bone cement was evaluated as an orbital implant for filling the anophthalmic cavity of dogs. The animals were from the Ophthalmology Unit of the Veterinary Hospital, Universidade Estadual Paulista - Jaboticabal, and from a private veterinary clinic. The study was conducted during two years. The patients' age ranged from 2-11 years old, and their weight from 9-50 kg. The implants ranged from 19 to $25 \mathrm{~mm}$ in diameter. Local edema was observed in all animals during the immediate postoperative period. Of the ten performed surgeries in seven animals, a satisfactory, aesthetic result was observed in nine (90\%) while in one (10\%) of them it was unsatisfactory due to complications that led to the implant removal. The use of the double-setting alpha-tricalcium phosphate bone cement to fill anophthalmic sockets is feasible and could be an alternative to improve the cosmetic appearance after eyeball removal.
\end{abstract}

Keywords: Hydroxyapatite. Enucleation eye. Artificial eye.

\section{Introdução}

A enucleação é a cirurgia orbitária mais comumente realizada em animais, sendo recomendada para casos de olho cego doloroso, em estágio final de glaucoma ou diabetes, e nos casos de endoftalmite ou trauma ocular severo (MOLTENO et al., 1973; McLAUGHLIN et al., 1995; JORDAN; BAWAZZER, 2001; CHEN; CUI, 2006; CHIN; MARGOLIN; FINGER, 2006; HEWES; KEOUGHAN; GUTIERREZNIBEYRO, 2007; PHAN; HWANG; McCULLEY, 2012; FOSSUM, 2013). Tal procedimento é também empregado no tratamento de tumores intraoculares, não passíveis de outras formas de terapia cirúrgica ou medicamentosa (HAMOR; ROBERTS; SEVERIN,

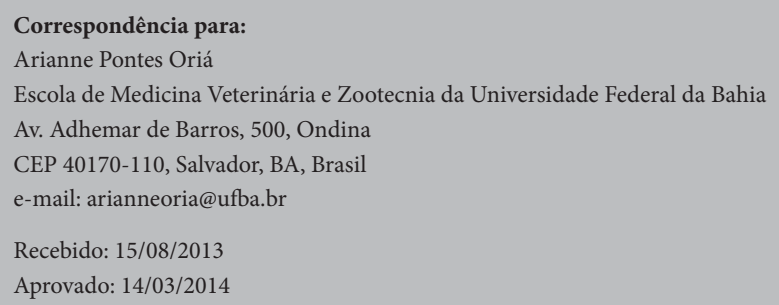


1993; JORDAN; BAWAZZER, 2001; CHIN; MARGOLIN; FINGER, 2006; HEWES; KEOUGHAN; GUTIERREZ-NIBEYRO, 2007; SPIESS, 2007; PHAN; HWANG; McCULLEY, 2012; FOSSUM, 2013). Tratase, portanto, da remoção cirúrgica do bulbo do olho, da membrana nictitante e sua glândula, de parte da conjuntiva e da porção distal da pálpebra superior e inferior, deixando o máximo possível de tecidos moles orbitais para minimizar a depressão orbital (FOSSUM, 2013). Já a exenteração, remoção do bulbo do olho, conteúdo orbital e porções das pálpebras, é indicada para pacientes com neoplasia orbital ou ocular, ou infecção na qual houve disseminação para além do bulbo do olho (SPIESS, 2007; FOSSUM, 2013).

Em cães, intercorrências no pós-operatório não são relevantes na enucleação, no entanto na exenteração são significativas. Deiscência de sutura, deslocamento traumático e rotação do implante, seroma orbital, hemorragia, infecção e extrusão são relatadas (SPIESS, 2007). Tanto a enucleação quanto a exenteração ocasionam uma órbita côncava e cosmeticamente inaceitável, principalmente em animais mesocefálicos e dolicocefálicos, os quais apresentam órbitas profundas (NASISSE et al., 1988). Para repor o vazio facial ocasionado por esses procedimentos cirúrgicos, compostos biologicamente inertes podem ser utilizados para melhoria da aparência estética (CHIN; MARGOLIN; FINGER, 2006; SPIESS, 2007; ARAF et al., 2010; BRANCO; GRUMANN JÚNIOR, 2012; MIYASHITA et al., 2013).

Os implantes não integráveis são os mais utilizados em Medicina Veterinária para o preenchimento de cavidades anoftálmicas. Estes não permitem a integração dos tecidos moles adjacentes, no entanto ensejam boa aparência estética (ORIÁ et al., 2011). O implante de silicone e o de polimetilmetacrilato (PMMA) são os rotineiramente utilizados em Medicina Veterinária (KOCH, 1981; NASISSE et al., 1988; HAMOR; ROBERTS; SEVERIN, 1993; TALIERI et al., 2004; SPIES, 2007; YI et al., 2009; ORIÁ et al., 2011; ORIÁ et al., 2012).
As esferas utilizadas neste trabalho foram confeccionadas a partir do cimento ósseo de alfa-fosfato tricálcico de dupla pega $(\alpha-\mathrm{TCP})$ desenvolvido por Santos (2002) e experimentado como implante intraocular em coelhos eviscerados por Oriá et al. (2006), nos quais se comportou de maneira inerte e não integrável. Este trabalho teve como objetivo reportar a utilização do $\alpha$-TCP como opção de implante para preenchimento de cavidade anoftálmica em cães.

\section{Materiais e Métodos}

Foram realizadas dez cirurgias com implante de esferas de $\alpha$-TCP na órbita de sete cães provenientes da rotina do Serviço de Oftalmologia do Hospital Veterinário “Governador Laudo Natel”, da Universidade Estadual Paulista - UNESP - Câmpus de Jaboticabal e de uma clínica privada, em um período de dois anos.

Quanto ao sexo, cinco (71\%) eram machos e dois (29\%), fêmeas. A idade dos pacientes variou de dois a 11 anos e, o peso entre nove e $50 \mathrm{~kg}$. Dos procedimentos realizados nos sete animais, três $(42,8 \%)$ foram enucleações bilaterais, três $(42,8 \%)$ foram enucleações unilaterais e um $(14,4 \%)$ foi exenteração. Entre as afecções apresentadas, encontram-se glaucoma crônico - três (43\%), perfuração corneal e prolapso do trato uveal - um (14\%) e neoplasia -três (43\%) (Tabela 1).

As esferas foram confeccionadas previamente e esterilizadas por autoclavagem a $132^{\circ} \mathrm{C}$, durante 20 minutos. Os implantes variaram de 19 a $25 \mathrm{~mm}$ de diâmetro. A seleção para o tamanho das esferas foi balizada na mensuração do diâmetro do bulbo do olho removido. Para os animais com olhos buftálmicos, devido ao glaucoma crônico, foi adotada a mensuração do diâmetro corneal do olho adelfo. Nos animais que apresentaram glaucoma crônico bilateral, adotou-se tamanho de esfera dois milímetros menor que o bulbo do olho. Contudo, durante o ato cirúrgico, também estavam disponíveis implantes de todos os tamanhos para que se pudesse escolher o que mais se adequava à órbita. 
Tabela 1 - Distribuição dos sete cães que receberam o implante do $\alpha$-TCP para preenchimento da cavidade anoftálmica após a enucleação do bulbo ocular - Jaboticabal - 2013

\begin{tabular}{|c|c|c|c|c|c|c|c|c|c|}
\hline A & Raça & $\mathrm{S}$ & $\mathbf{P}$ & $\mathrm{I}$ & Afecção oftálmica & TI & PA & RE & Complicações pós-cirúrgicas \\
\hline 1 & Boxer & $\mathrm{F}$ & 23 & 2 & Melanoma intraocular - bilateral & 23 & $6 \mathrm{~m}$ & $S$ & Morte por metástase \\
\hline 2 & SRD & $\mathrm{M}$ & 30 & 8 & Glaucoma crônico - bilateral & 23 & $18 \mathrm{~m}$ & S & SI \\
\hline 3 & Shitzu & M & 9 & 7 & $\begin{array}{l}\text { Perfuração corneana, prolapso de } \\
\text { trato uveal - olho esquerdo }\end{array}$ & 19 & $4 \mathrm{~m}$ & S & SI \\
\hline 4 & São Bernardo & M & 50 & 3 & Glaucoma crônico - bilateral & 25 & $3 \mathrm{~m}$ & S & SI \\
\hline 5 & Beagle & $\mathrm{F}$ & 12 & 10 & $\begin{array}{l}\text { Carcinoma de células escamosas em } \\
\text { pálpebra superior e inferior com } \\
\text { infiltração para conjuntiva bulbar - } \\
\text { olho direito }\end{array}$ & 22 & $10 \mathrm{~d}^{*}$ & S & SI \\
\hline 6 & SRD & M & 15 & 11 & $\begin{array}{l}\text { Glaucoma crônico e ulceração } \\
\text { corneana - olho esquerdo }\end{array}$ & 22 & $20 \mathrm{~d}$ & NS & $\begin{array}{l}\text { Exposição do implante e infecção } \\
\text { após } 10 \text { dias. Optou-se pela } \\
\text { remoção do implante }\end{array}$ \\
\hline 7 & SRD & M & 13 & 7 & $\begin{array}{l}\text { Neoplasia" retrobulbar - } \\
\text { olho esquerdo }\end{array}$ & 22 & $10 \mathrm{~m}$ & S & SI \\
\hline
\end{tabular}

Legenda: A animal; $\mathbf{S}$ sexo; P peso em kg ; I idade; TI tamanho do implante em milímetros; PA período de avaliação; $\mathbf{m}$ meses; $\mathbf{d}$ dias; RE resultado estético; SRD sem raça definida; $\mathbf{F}$ fêmea; $\mathbf{M}$ macho; $\mathbf{S}$ satisfatório; NS não satisfatório; SI sem intercorrências. \# Exame histopatológico não foi realizado por dificuldade financeira. ${ }^{*}$ Perdeu-se o contato com o proprietário. $\alpha$-TCP $=$ cimento de alfa-fosfato tricálcico de dupla pega

Para o procedimento cirúrgico, os animais foram submetidos à anestesia geral inalatória. Posteriormente à tricotomia da região periorbital seguiu-se a antissepsia dos fórnices conjuntivais e de toda a superfície ocular. Continuou-se o preparo da superfície cutânea periocular com PVP-I a $10 \%$ e foi colocado o campo operatório. A fim de facilitar as manobras cirúrgicas, foi realizada cantotomia lateral. Ato contínuo, conduziu-se a enucleação do bulbo do olho pela técnica subconjuntival lateral ou a exenteração (SPIESS, 2007; FOSSUM, 2013). Seguiu-se a introdução manual da esfera de cimento de $\alpha$-TCP na cavidade orbitária, circundada por fáscia orbitária e remanescentes dos músculos extraoculares previamente transeccionados, com pontos simples interrompidos com fio poliglactina 910 (Vicryl $^{\circledR} 4 / 0,3 / 0,2 / 0$ ou 0, Ethicon Johnson \& Johnson, São Paulo - Brasil). No caso do animal número 5 , como havia infiltração tumoral para a conjuntiva bulbar, o implante foi mantido no interior da cavidade orbitária por quatro suturas simples ancoradas no periósteo, uma vez que se procedeu à remoção da fáscia orbitária e remanescentes dos músculos extraoculares (Figura 1). O espaço morto foi reduzido com fio poliglactina 910 , em padrão contínuo simples, e a dermorrafia com fio de poliamida
(Mononylon ${ }^{\circledast} 3 / 0$ ou 2/0, Ethicon Johnson \& Johnson, São Paulo - Brasil), em padrão simples interrompido. Colar elizabetano foi utilizado até a remoção dos pontos, de sete a dez dias após a cirurgia.

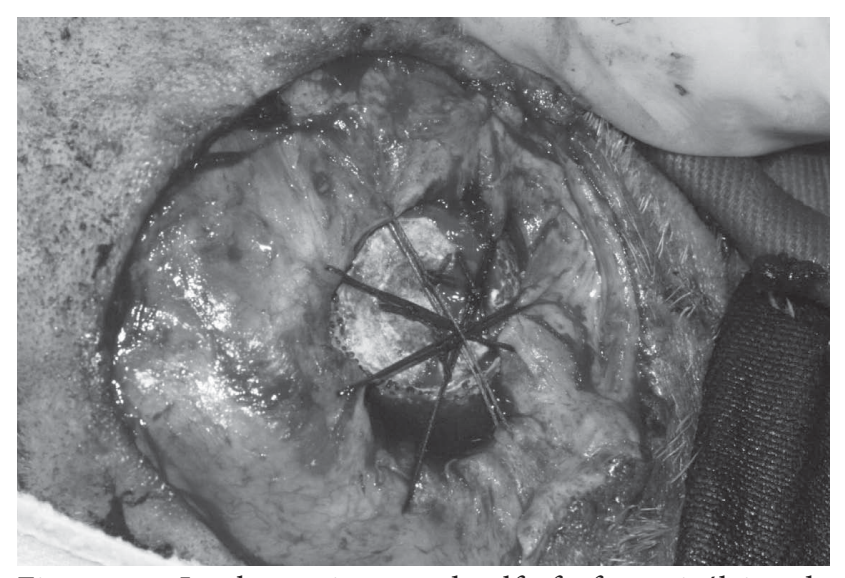

Figura 1 - Implante cimento de alfa-fosfato tricálcico de dupla pega em órbita de cão. Notar a manutenção do implante no interior da cavidade orbitária por quatro suturas simples ancoradas no periósteo

Fonte: (ORIÁ, 2006)

Adotou-se como antibiótico cefalexina (Cefalexina ${ }^{\bowtie}$, SEM), na dose de $25 \mathrm{mg} / \mathrm{kg}$, a cada 12 horas, por via oral, durante sete dias. Adicionalmente, foram administrados meloxicam (Maxicam ${ }^{\oplus}$, Ouro Fino, São Paulo, Brasil), na dose de $0,1 \mathrm{mg} / \mathrm{kg}$, a cada 24 horas, 
por via oral, durante cinco dias, e buprenorfina (Tengesic $^{\oplus}$, Shering Plough, Rio de Janeiro, Brasil), na dose de $0,01 \mathrm{mg} / \mathrm{kg}$, em aplicação única no pós-operatório imediato. Foi solicitada a higienização diária do local da sutura com gaze estéril embebida em solução fisiológica, seguida da utilização de iodo povidine.

As avaliações pós-operatórias foram efetuadas mediante exame clínico no pós-operatório imediato e após sete a dez dias, durante a retirada dos pontos (Figura 2a). Considerou-se o resultado satisfatório quando o aspecto estético foi aceitável, com a ausência de secreção e a completa cicatrização da ferida cirúrgica (Figura 2b).

\section{Resultados e Discussão}

No presente trabalho, a enucleação foi empregada como método de tratamento para alterações orbitárias, fato já descrito por Molteno et al. (1973); Hamor, Roberts e Severin (1993); McLaughlin et al. (1995); Jordan e Bawazzer (2001); Chen e Cui (2006); Chin, Margolin e Finger (2006); Hewes e Keoughan e Gutierrez-Nibeyro (2007); Spiess (2007); Phan, Hwang e McCulley (2012); Fossum (2013), que indicaram o procedimento como método efetivo para o manejo de olhos cegos e dolorosos, por glaucoma incontrolável, endoftalmites, trauma ocular severo com hemorragia e como opção para o tratamento de tumores intraoculares. A exenteração foi realizada no paciente número 5 conforme indicação de Spiess (2007) e Fossum (2013) para o tratamento de neoplasias que envolvem o bulbo do olho e os anexos oftálmicos.

Implantes de PMMA compactos podem ser utilizados para o preenchimento de cavidades anoftálmicas resultantes de enucleações, eviscerações ou exenterações, principalmente devido ao baixo custo (RAHAL; BERGAMO; ISHIY, 2000; TALIERI et al., 2004; ORIÁ et al., 2011; ORIÁ et al., 2012; MIYASHITA et al., 2013), no entanto, devido à elevada adesão bacteriana que o implante proporciona, algumas complicações podem ocorrer durante seu uso, como favorecimento da infecção, exposição do implante, extrusão espontânea e inflamação crônica (NASISSE, 1988; EPPLEY, 2003). Assim, torna-se necessária a busca por novos biomateriais para compor os implantes.

A remoção do bulbo ocular nem sempre é aceita pelo proprietário de pequenos animais, uma vez que a cosmética é geralmente sua maior preocupação (HAMOR et al., 1994). Essa questão foi levantada pelos proprietários no momento da consulta, no entanto todos ficaram satisfeitos com o resultado pós-operatório mesmo

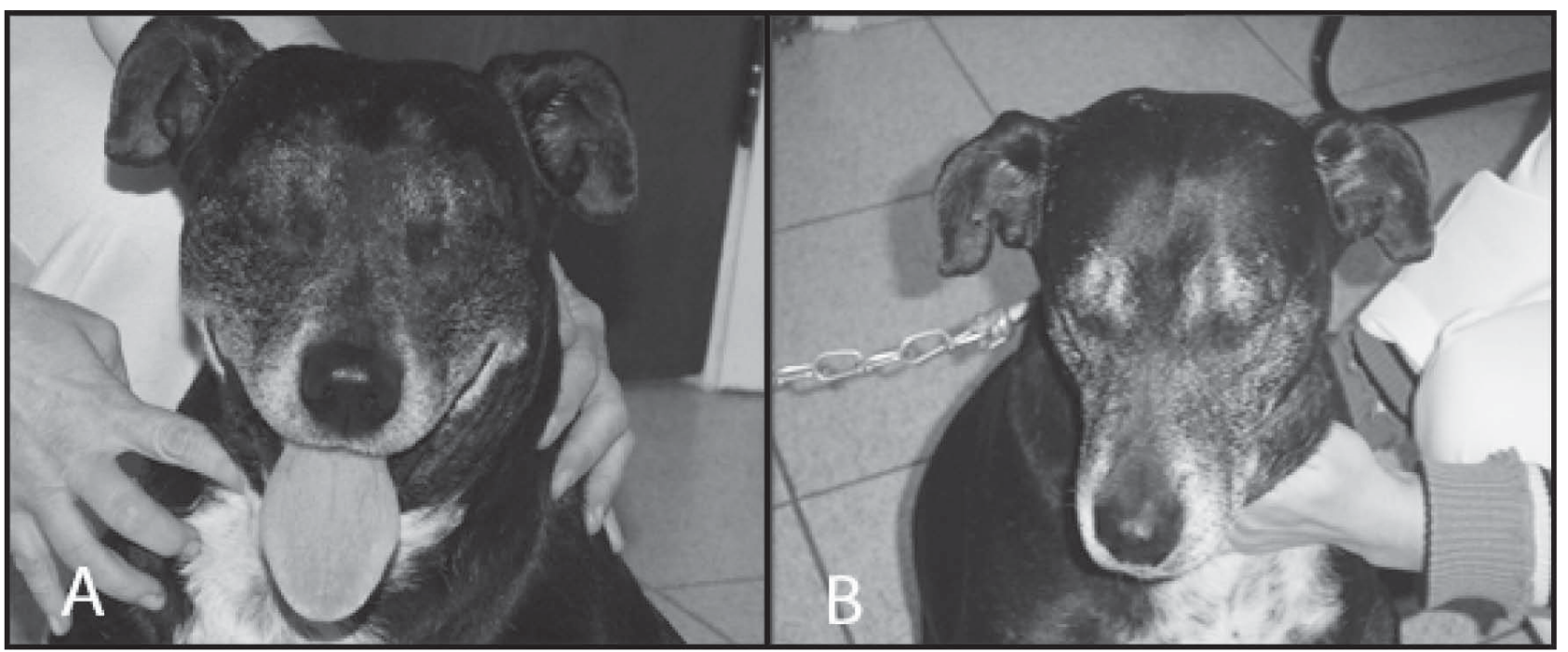

Figura 2 - Imagem fotográfica do animal 2, 15 dias (A) e 18 meses (B) após a colocação do implante cimento de alfafosfato tricálcico de dupla pega na cavidade anoftálmica. Notar a ausência de depressão orbitária

Fonte: (ORIÁ, A-2003; B-2005) 
no caso em que o implante teve de ser retirado. Os resultados estéticos alcançados com o cimento foram semelhantes aos obtidos com a utilização de PMMA por outros pesquisadores (NASISSE et al., 1988; HAMOR; ROBERTS; SEVERIN, 1993; SPIESS, 2007).

Das dez cirurgias realizadas em sete animais, em nove o resultado estético foi satisfatório, enquanto em um foi insatisfatório devido a complicações que levaram à remoção do implante. Os animais apresentaram conforto e ausência de sinais que pudessem sugerir dor, como sonolência, hipoatividade e relutância à manipulação local, conforme observação clínica e relato dos proprietários. Fato observado inclusive nos animais que receberam o implante bilateralmente.

A utilização de implante com tamanho adequado facilitou a aposição do tecido orbitário sobre a superfície do implante (HAMOR; ROBERTS; SEVERIN, 1993), o que minimizou a possibilidade de deslocamento ou extrusão, ao passo que nenhum dos animais apresentou tal complicação durante o período de estudo. Ressalta-se que o diâmetro dos implantes em cães varia de 12 a 28 mm (HAMOR et al., 1994; SLATER; BASHER, 2007) e deve ser determinado pela profundidade e diâmetro do defeito na órbita. No presente trabalho, foram utilizados implantes de 19 a $25 \mathrm{~mm}$ de diâmetro, os quais, antes de serem recobertos pelos tecidos remanescentes, foram inseridos na cavidade orbitária para a verificação da proporcionalidade do preenchimento. Não foi necessária a mudança de tamanho de nenhum dos implantes mensurados previamente ao ato cirúrgico.

A utilização de implantes com o intuito de melhorar o aspecto estético após cirurgias mutilantes envolvendo o bulbo ocular é comumente realizada na medicina para evitar o desenvolvimento de distúrbios psicológicos que culminem em depressão (MIGLIORI, 2002; RAIZADA; RANI, 2007; BRANCO; GRUMANN JÚNIOR, 2012). Tal procedimento é realizado mesmo na presença de processos neoplásicos agressivos, enumerando-se poucas complicações como a deformidade do sulco superior, proptose e exotropia (McLAUGHLIN et al., 1995; CHIN; MARGOLIN; FINGER, 2006).

Três dos sete animais $(42,8 \%)$ eram portadores de neoplasias, sendo intraocular (animal 1), em anexos oftálmicos (animal 5) e retrobulbar (animal 7). Em todos os animais, a ultrassonografia abdominal e as radiografias torácicas não revelaram a presença de metástases. O paciente número 1 foi submetido à enucleação por apresentar olhos cegos e com intenso desconforto (glaucomatosos com presença de massas em trato uveal). Ao exame histopatológico, identificou-se a neoformação como melanoma intraocular com envolvimento de íris, corpo ciliar e esclera, em ambos os olhos. O proprietário foi orientado sobre as complicações e a elevada possibilidade de metástase, fato este que levou o animal à eutanásia seis meses após a cirurgia. $\mathrm{O}$ paciente número 5 apresentava massas ulceradas na região periocular, envolvendo ambas as pálpebras, com infiltração para conjuntiva bulbar. Depois da exenteração, o exame histopatológico definiu a alteração como carcinoma de células escamosas. Devido ao fato de o proprietário residir em outra cidade, o contato com o paciente foi perdido dez dias após a cirurgia, momento em que foram retirados os pontos. No caso do animal número 7 o paciente apresentava massa proeminente dorsal ao olho. Ao exame ultrassonográfico, foi constatada a presença de neoformação na região retrobulbar da órbita esquerda. Devido à limitação financeira do proprietário, o exame histopatológico não pode ser realizado. $\mathrm{O}$ animal foi acompanhado regularmente (a cada 30 dias) para identificação de recidivas com o exame clínico e não apresentou sinais de alterações após dez meses da cirurgia, momento em que foi concluída a pesquisa.

Apesar de haver resistência quanto à utilização de implantes em indivíduos portadores de neoplasias oculares, independente do tipo, devido à possibilidade de recidivas e pelo favorecimento da rejeição do implante (McLAUGHLIN et al., 1995), o uso das esferas de $\alpha$-TCP mostrou-se factível no presente trabalho. Poucas são as descrições de utilização de implantes em pacientes veterinários portadores de neoplasias 
oculares. Talieri et al. (2004) utilizaram o polimetilmetacrilato em pasta na cavidade orbitária de três cães exenterados devido à neoplasia intraocular, orbital e palpebral, e Naranjo e Dubielzig (2013), que ao realizarem o estudo retrospectivo em cães e gatos portadores de neoplasias oculares, submetidos à evisceração com inserção de implante, notaram que a reincidência do processo neoplásico, devido à permanência de resquícios uveais, foi a maior causa de fator complicador observada. Saliente-se, portanto, que as complicações da utilização de implantes em pacientes portadores de neoplasias oculares deverão ser informadas aos proprietários dos animais para que a decisão pelo uso do implante seja tomada de forma consciente.

A malha de prolene ou dacron pode ser utilizada ancorada ao periósteo da margem orbitária com o intuito de reduzir a depressão que ocorre após a exenteração (SPIESS, 2007). No animal número 5, para minimizar a possibilidade de extrusão ou deslocamento do implante após a exenteração, por falta de uma malha biocompatível, foram utilizadas quatro suturas simples ancoradas no periósteo.

Em todos os animais, conforme desejado, houve uma discreta proeminência na região orbitária no local onde o implante foi inserido. A principal complicação foi a lesão cutânea com exposição do implante, associada à infecção secundária em um animal (10\% das cirurgias). As complicações são incomuns, mas podem ser observados; edema, hemorragia, infecção da ferida cirúrgica, enfisema orbitário, formação de secreções por remanescentes de tecido glandular, drenagem de sangue ou secreção serosa nas narinas por patência dos ductos nasolacrimais e fistulação (NASISSE et al., 1988; SPIESS, 2007). No caso do animal número 6 , o proprietário relatou ter retirado o colar e que ocorreu traumatismo sete dias após a cirurgia. Três dias depois, houve a exposição do implante e foram notados sinais de infecção, momento em que foi retirada a esfera implantada, realizado o debridamento tecidual e ocorreu a cicatrização adequada. Nenhuma outra intercorrência foi notada, exceto o edema local presente em todos os pacientes durante o pósoperatório imediato e que perdurou por 72 a 96 horas.

A exposição do implante de hidroxiapatita natural, quando utilizado em humanos, ocorre por infecção, tensão na sutura, tamanho exagerado da esfera, localização superficial e inadequada do implante e falha técnica (GOLDBERG; HOLDS; EBRAHIMPOUR, 1992). No presente trabalho, as complicações podem ter sido relacionadas a questões como falha técnica, trauma com exposição do implante e infecção secundária, que podem ter sido agravadas pelo formato esférico do implante.

A melhoria do efeito cosmético e a diminuição da possibilidade de rotação do implante podem ser alcançadas mediante a remoção do seguimento anterior, face rostral da esfera, de maneira a ser obtida uma superfície reta (SPIESS, 2007). Possivelmente, além das melhorias, a confecção nesse formato também poderia minimizar traumas e, consequentemente, diminuição do risco de exposição das esferas com posterior extrusão.

Gatos e cavalos apresentam maiores índices de extrusão de implantes comparativamente aos cães (HAMOR; ROBERTS; SEVERIN, 1993). NASISSE et al. (1988), ao acompanharem cinco gatos receptores de implantes de PMMA, observaram que três apresentaram acúmulo de fluido orbital, considerado como o principal fator de insucesso, e que das 73 esferas de PMMA implantadas na órbita de cães, apenas três apresentaram complicações, cujas causas foram o deslocamento do implante durante briga, a extrusão espontânea e a inflamação crônica.

O cimento mostrou-se satisfatório no preenchimento da órbita de cães sob o ponto de vista cosmético e de biocompatibilidade. Ainda não há um consenso quanto ao material ideal a ser utilizado como implante oftálmico, por este motivo, outras investigações deverão ser conduzidas em busca deste objetivo.

A utilização do cimento de alfa-fosfato tricálcico de dupla pega no preenchimento de cavidade anoftálmica mostrou-se factível e é uma alternativa para melhorar a aparência estética após a enucleação de cães. 


\section{Referências}

ARAF, D.; ASSAE, O. M.; BRITO, R. V.; AQUINO JÚNIOR, G; SILVA, T. A. G. Implante orbital misto para reconstrução de cavidade anoftálmica: relato de caso. Arquivo Brasileiro de Oftalmologia, v. 73, n. 1, p. 81-83, 2010.

BRANCO, F. C. E.; GRUMANN JÚNIOR, A. Perfil dos pacientes submetidos à reconstrução primária da cavidade orbitária com implante de Mules após enucleação e evisceração. Arquivo Brasileiro de Oftalmologia, v. 71, n. 4, p. 221-225, 2012.

CHEN, Y.; CUI, H. High density porous polyethylene material (Medpor) as an unwrapped orbital implant. Journal of Zhejiang University-SCIENCE B., v. 8, n. 7, p. 679-682, 2006.

CHIN, K.; MARGOLIN, C. B.; FINGER, P. T. Early ocular prosthesis insertion improves quality of life after enucleation. Optometry, v. 77, n. 2, p. 71-75, 2006.

EPPLEY, B. L. Alloplastic cranioplasty. Operative Techniques in Plastic and Reconstructive Surgery, v. 9, n. 1, p. 16-22, 2003.

FOSSUM, T. W. Surgery of the eye. In: FOSSUM, T. W. Small animal surgery. 4. ed. St. Louis: Elsevier, 2013. p. 289-324.

GOLDBERG, R. A.; HOLDS, J. B.; EBRAHIMPOUR, J. Exposed hydroxyapatite orbital implants. Ophthalmology, v. 99, n. 5, p. 831-836, 1992.

HAMOR, R. E.; ROBERTS, S. M.; SEVERIN, G. A. Use of orbital implants after enucleation in dogs, horses, and cats: 161 cases (1980-1990). Journal of the American Veterinary Medical Association, v. 203, n. 5, p. 701-706, 1993.

HAMOR, R. E.; WHITLEY, R. D.; McLAUGHLIN, S. A.; LINDLEY, D. M.; ALBERT, R. A. Intraocular silicone prostheses in dogs: a review of the literature and 50 new cases. Journal of the American Animal Hospital Association, v. 30, p. 66-69, 1994.

HEWES, C. A.; KEOUGHAN, G. C.; GUTIERREZ-IBEYRO, S. Standing enucleation in the horse: a report of 5 cases. Canadian Veterinary Journal, v. 48, p. 512-514, 2007.

JORDAN, D. R.; BAWAZZER, A. Experience with 120 synthetic hydroxyapatite implants (FCI3). Ophthalmic Plastic and Reconstructive Surgery, v. 17, n. 3, p. 184-190, 2001.

$\mathrm{KOCH}, \mathrm{S}$. A. Intraocular prosthesis in the dog and cat: the failures. Journal of the American Veterinary Medical Association, v. 179, n. 9, p. 883-885, 1981.

McLAUGHLIN, S. A.; RAMSEY, D. T.; LINDLEY, D. M.; GILGER, B. C.; GERDING, P. A.; WHITLEY, R. D. Intraocular silicone prosthesis implantation in eyes of dogs and a cat with intraocular neoplasia: nine cases (1983-1994). Journal of the American Veterinary Medical Association, v. 207, n. 11, p. 1441-1443, 1995.

MIGLIORI, E. M. Enucleation versus evisceration. Current Opinion in Ophthalmoly, v. 13, n. 5, p. 298-302, 2002.

MOLTENO, A. C. B.; VAN RENSBERG, J. H. J.; VAN ROOYEN, B.; ANCKER, E. "Physiologica" orbital implant. The British Journal of Ophthalmology, v. 57, n. 615, p. 615-621, 1973.

MIYASHITA, D.; CHAHUD, F; BARROS, S. G. E.; ALBUQUERQUE, V. B.; GARCIA, D. M.; CRUZ, A. A. V. Tissue ingrowth into perforated polymethymethacrylatel orbital implants: an experimental study. Ophthalmic Plastic and Reconstructive Surgery, v. 29, n. 3, p. 160-163, 2013.

NARANJO, C.; DUBIELZIG, R. R. Histopathological study of the causes for failure of intrascleral prostheses in dogs and cats. Veterinary Ophthalmology, p. 1-8, 2013. In press.

NASISSE, M. P.; VAN EE, R. T.; MUNGER, R. J.; DAVIDSON, M. G. Use of methyl methacrylate orbital prostheses in dogs and cats: 78 cases (1980-1986). Journal of the American Veterinary Medical Association, v. 192, n. 4, p. 539-542, 1988.

ORIÁ, A. P.; NETO, F. A.; LAUS, J. L.; SANTOS, L. A.; PIZA, E. T.; BRUNELLI, A. T.; NISHIMORI, C. T.; SOUZA, A. L. Evaluation of a double-setting alfa-tricalcium phosphate cement in eviscerated rabbit eyes. Ophthalmic Plastic and Reconstructive Surgery, v. 22, n. 2, p. 126-130, 2006

ORIÁ, A. P.; DÓREA NETO, F. A.; GOMES JUNIOR, D. C.; PINNA, M. H.; LAUS, J. L. Utilização de implantes para o manejo de cavidades anoftálmicas. Medicina Veterinária, v. 5, n. 4, p. 1-12, 2011.

ORIÁ, A. P.; DÓREA NETO, F. A.; SANTOS, L. A.; PIZZA, E. T.; BRUNELLI, A. T. J.; NISHIMORI, C. T.; SOUZA, A. L. G.; GOMES JUNIOR, D. C.; LAUS, J. L. Evaluation of polymethylmethacrylate as ocular implant in rabbits subjected to evisceration. Revista Ceres, v. 59, n. 4, p. 452-457, 2012.

PHAN, L. T.; HWANG, T. N.; McCULLEY, T. J. Evisceration in the modern age. Middle East African Journal of Ophthalmology, v. 19, n. 1, p. 24-33, 2012.

RAHAL, S. C.; BERGAMO, F. M. M.; ISHIY, H. M. Prótese intraocular de resina acrílica em cães e gatos. Arquivo Brasileiro de Medicina Veterinária e Zootecnia, v. 52, n. 4, p. 319-324, 2000.

RAIZADA, K.; RANI, D. Ocular prosthesis. Contact Lens \& Anterior Eye, v. 30, n. 3, p. 152-162, 2007.

SANTOS, L. A. dos. Desenvolvimento de cimento de fosfato de cálcio reforçado por fibras para o uso na área médicoodontológica. 2002. 275 f. Tese (Doutorado em Engenharia de Biomateriais) - Faculdade de Engenharia Mecânica, Universidade Estadual de Campinas, Campinas, 2002.

SLATER, D.; BASHER, B. Órbita. In: SLATTER, D. Manual de cirurgia de pequenos animais. 3. ed. São Paulo: Manole, 2007. p. $1430-1454$

SPIESS, B. M. Diseases and surgery of the canine orbit. In: GELATT, K. N. Veterinary ophthalmology. 4. ed. Ames: Blackwell, 2007. p. 539-562.

TALIERI, I. C.; BUQUERA, L. E. C.; ORIÁ, A. P.; BRUNELLI, A. T. J.; ALMEIDA, D. E.; LAUS, J. L. Use of methyl methacrylate, moulded in its paste phase, to fill the orbital cavity of dogs, after exenteration in cases of ophthalmic neoplasias: a report of three cases. Ciência Rural, v. 34, n. 2, p. 567-571, 2004.

YI, N. A.; PARK, S. A.; JEONG, M. B.; KIM, W. T.; KIM, S. E.; KIM, J. Y.; CHAE, J. M.; JANG, K. J.; SEONG, J. K.; SEO, K. M. Comparison of orbital prosthesis motility following enucleation or evisceration with sclerotomy with or without a motility coupling post in dogs. Veterinary Ophthalmology, v. 3, n. 12, p. 139-151, 2009. 